\title{
Assessment of dynamic mechanical behaviour of reinforced concrete beams using a blast simulator
}

\author{
Marco Peroni ${ }^{\text {a }}$, George Solomos, Alessio Caverzan, Martin Larcher, and Georgios Valsamos \\ European Commission Joint Research Centre (JRC), Institute for the Protection and Security of the Citizen (IPSC), \\ European Laboratory for Structural Assessment (ELSA), via E. Fermi 2749, 21027 Ispra (VA), Italy
}

\begin{abstract}
Critical infrastructures may become the target of terrorist bombing attacks or may have to withstand explosive loads due to accidents. The impulsive load connected to explosions is delivered to the structure in a few milliseconds forcing it to respond or fail in a peculiar mode. With reference to the above scientific framework this work presents an innovative apparatus designed and developed at the European Laboratory for Structural Assessment to reproduce a blast pressure history without using explosives. This apparatus is practically a hybrid nitrogen-spring-driven actuator that accelerates masses of up to $100 \mathrm{~kg}$ to a maximum velocity of about $25 \mathrm{~m} / \mathrm{s}$ that impact against the tested structure. The pressure-load history applied to the structure is modulated and reshaped using appropriate layers of elastic soft materials (such as polymeric foams) placed between the specimen and the impacting masses. Specific instrumentation has extensively been utilised to investigate the blast simulator performance and to precisely measure the pressure loads applied to the specimen. A series of tests on real scale reinforced concrete beams/columns $(250 \times 250 \times 2200 \mathrm{~mm})$ has been performed to efficiently assess the performance and potentiality of the new blast simulator. Results are under evaluation. In addition to the experimental work, a series of numerical simulations by means of the explicit FEM code EUROPLEXUS have been carried out to support and improve the equipment design.
\end{abstract}

\section{Introduction}

Critical infrastructures in fields such as energy, health, communication, government, transport etc. are made of physical structures, or are housed in physical structures. Such structures may naturally become the target of terrorist bombing attacks. Measures to protect them will certainly be taken, involving prevention, intelligence, detection, deterrence etc., but if everything fails, it is very important that the mechanical structure itself mitigates some effects of the explosion and maintains certain functionalities.

A typical pressure wave curve (which eventually will load a structure) at some distance from an explosion is shown in Fig. 1. Its main characteristics concerning damaging effects on structures are the magnitude of the overpressure, the duration of the positive phase and especially its positive impulse, i.e., the area under the curve over the positive phase. This impulsive load will be delivered to a structure in a few milliseconds forcing it to respond or fail in a peculiar mode. This necessitates that models and design techniques for blast resistant structures be thoroughly validated with reliable data from field tests. However, such tests with actual explosions are expensive, they are usually performed within military grounds, and are often not easily reproducible. Thus, alternative testing methods are desirable, and this has been the case at the University of California in San Diego, where the first blast simulator facility has been built (2006).

As claimed, the effects of explosions are generated without the use of explosive materials. The facility produces repeatable, controlled blast load simulations on

\footnotetext{
${ }^{a}$ Corresponding author: marco.peroni@jrc.ec.europa.eu
}

full-scale columns and other structural components [1]. The simulator recreates the speed and force of explosive shock waves through servo-controlled hydraulic actuators that punch properly the test specimens.

With the ongoing work a similar blast simulation capability is under development within the EU by the JRC-ELSA, where long and strong experience with servocontrolled actuators resides. Concerning the currently required fast actuators, an alternative design concept has been implemented, which is believed to be capable of generating impacting loads resembling closer those of the real explosions of Fig. 1. This could allow the realistic testing of components to "simulated" explosions and would provide the necessary data for the validation of the computer tools.

The development of this technology will be important both for the research and the practicing engineers and architects who need design rules and guidelines. Besides characterizing blast effects on structural systems, the methodology will contribute to evaluating technologies for hardening and retrofitting buildings and bridges against terrorist bomb attacks. Further, it will help in the investigation of the problem of progressive collapse, i.e. the phenomenon where a local failure propagates in a disproportionate manner to lead to global failure, like the building collapse in the Oklahoma City bombing.

\section{Experimental setup - instrumentation}

The blast simulator is presently foreseen to be a small pneumatic/hydraulic facility and the sketch below summarizes the main parts of the apparatus (Fig. 2).

This is an Open Access article distributed under the terms of the Creative Commons Attribution License 4.0, which permits unrestricted use, distribution, and reproduction in any medium, provided the original work is properly cited. 


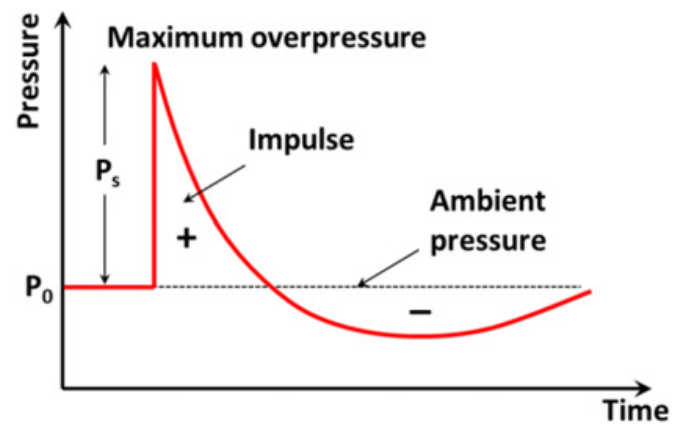

Figure 1. Pressure curve characteristics in free-air explosions.

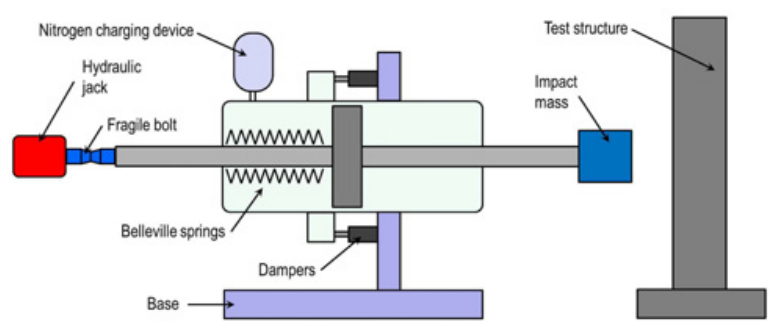

Figure 2. Sketch of blast simulator.

The operating principle of the testing rig is quite simple: the shaft is pulled through a fragile bolt by a hydraulic jack and this action compresses a series of Belleville springs inside the blast actuator. At this point there is the option to also raise the pressure inside the active chamber of the blast actuator by charging it with nitrogen (maximum pressure 100 bar). By continuing the pulling of the shaft (or, by increasing the nitrogen pressure) the bolt suddenly breaks and the piston and shaft rapidly accelerate pushing the impacting mass, which is attached at the other extreme of the shaft. When the piston has done most of its stroke, it starts to decelerate and the impacting mass is detached from it and collides with the tested structure reproducing local pressures similar to those of a blast wave. The implementation of this operating principle and details of the equipment can be found in [2] together with some preliminary tests. The structural elements tested in this work are two horizontally placed, simply supported beams of reinforced concrete.

The setup is similar to a dynamic three point bending, where the dynamic loading is applied over an area on the central portion of the beam, as shown in Figs. 3 and 4.

The two horizontally reacting aluminium supports have a semi-cylindrical geometry and are connected through a bar to the Reaction Wall (a very stiff concrete structure). The beam weight is vertically supported at two points by means of two Teflon sliders, which reduce horizontal friction during the test. The frame that supports the beam and the sliders is a modular structure made of aluminium profiles as is also the structure that supports the two linear bearing guides of the impacting mass. This frame allows a complete optical accessibility to the specimen from the top, where a high speed camera is placed.

The impact mass, composed of several parts, is designed to apply a pressure history similar to that of Fig. 1 and at the same time to effectively measure

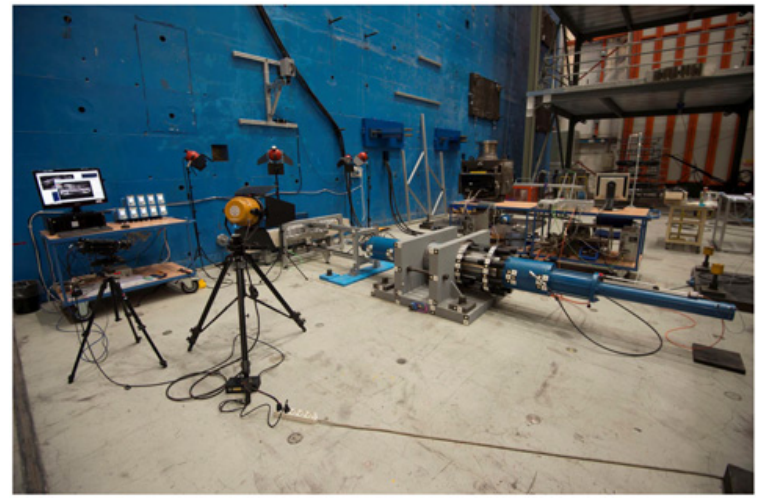

Figure 3. Final setup for blast experiments on RC beams.

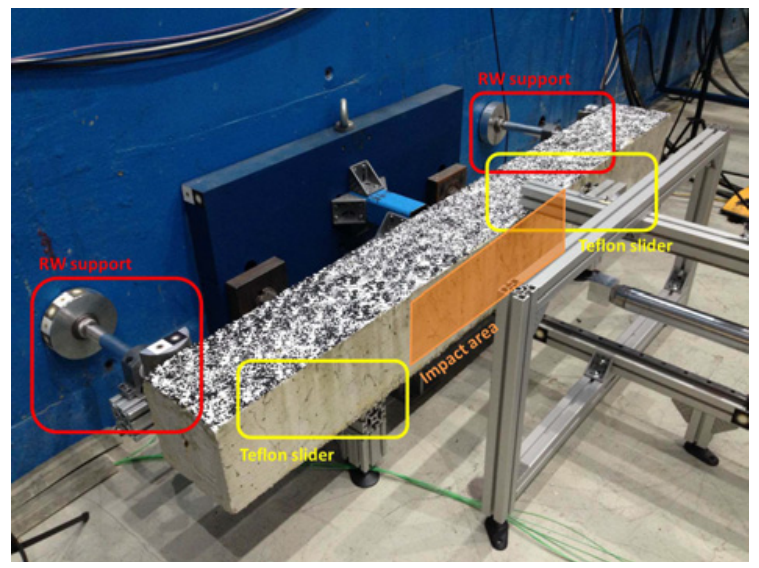

Figure 4. Blast simulator: specimen supports.

the contact forces between mass and specimen. This is essentially achieved by combining an instrumented mass composed of some rigid, light plates, in the front, connected through some load cells to the heavy, main mass behind (stainless steel). In this concept the plate masses (negligible compared to the stainless steel mass) do not affect the load cells results and the force signals are directly measured and not derived from calculations.

Specifically, the configuration tested in this campaign is shown in Fig. 5. The block of the impacting mass is composed of three aluminium plates $(290 \times 250 \times$ $10 \mathrm{~mm}$ ) connected with three independent piezoelectric load cells to a heavy stainless steel prismatic mass $(75 \times 75 \times 850 \mathrm{~mm})$. In front of each plate a layer of polyurethane foam has been placed to smooth the pressure pulse and reproduce closer the blast pressure history. The mass has been designed to slide on two linear bearing carriages and to rotate around two bearings rigidly connected to the carriages. These degrees of freedom reduce drastically the forces transmitted to the guiding rails, improving the safety and the lifetime of the equipment.

After the first test, two additional springs have been added (Fig. 5), in order to control rotations and avoid possible misalignment of the impacting mass during its free movement before the impact. The impactor, in its current design, can reliably measure a maximum load of $1980 \mathrm{kN}$, a suitable value also for impacting velocity greater than $15 \mathrm{~m} / \mathrm{s}$. 


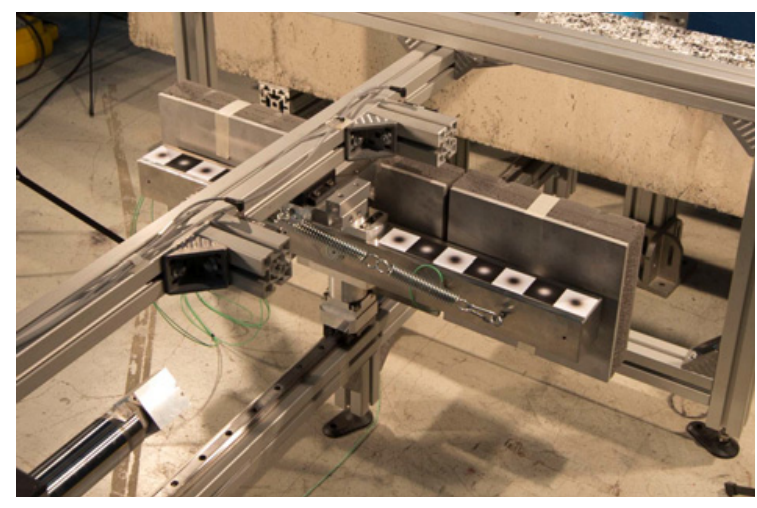

Figure 5. Blast simulator: impacting mass.

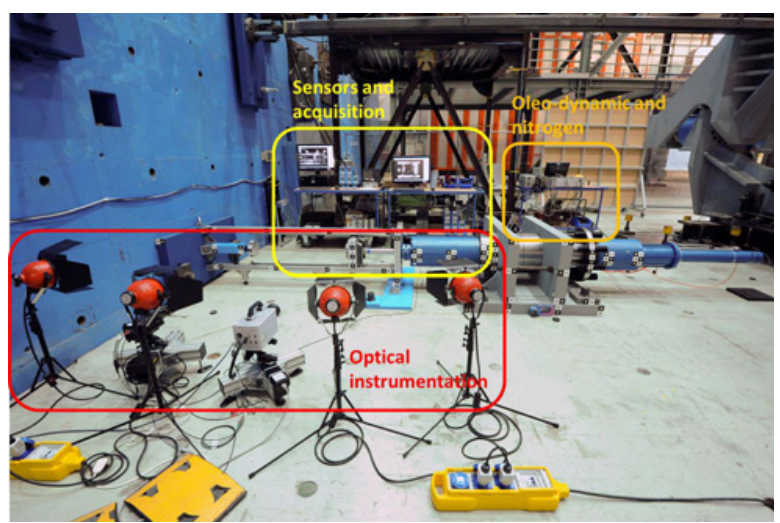

Figure 6. Blast simulator: instrumentation.

The instrumentation (electronic components and transducers) and the adopted devices are described below [2]. Figure 6 shows an example of typical instrumentation adopted in a blast simulator test.

The actuator is a servo-hydraulic axis electronically controlled with an in-house control system based on EtherCAT technology. In the control zone all components concerning the nitrogen booster have been placed to have a complete overview of actuator power devices. For what concerns the test instrumentation all sensors and transducers are acquired with a high speed transient recorder. The transducers typically adopted are piezoelectric accelerometers, load cells and pressure sensors and a series of photoelectric cells. In addition, two high speed cameras are normally used, synchronized to the transient recorder. The detailed list of instrumentation adopted and deployed during the experiments carried out is given below.

- 2 acquisition boards GAGE Octopus of 8 channels each with $20 \mathrm{Msample/s} \mathrm{per} \mathrm{channel.} \mathrm{Considering} \mathrm{the}$ test duration, a sampling frequency of $200 \mathrm{kHz}$ has been applied (pre-trigger 10000 points, post-trigger 100000 points).

- 1 High Speed camera IDT Y4 with 14 mm Nikkor lens. It films laterally the evolution of the whole experiment at a frequency of $800 \mathrm{fps}$ (pre-trigger 800 frames, posttrigger 800 frames).

- 1 High speed camera Photron SA1 with $21 \mathrm{~mm}$ Zeiss Distagon lens. It films the upper face of the specimen and impactor, for optical/ photogrammetric analysis

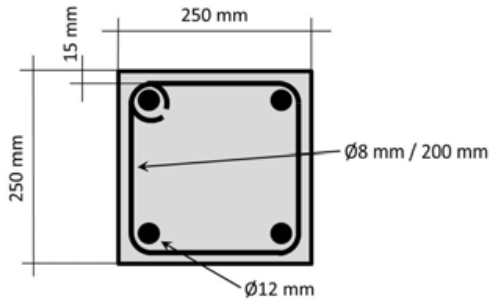

Figure 7. Reinforcement of the RC beams.

purposes, at a frequency of $5000 \mathrm{fps}$ (pre-trigger 0 frames, post-trigger 5000 frames).

- 9 Charge Amplifiers Kistler 5015 for the conditioning of piezoelectric sensors.

- 2 Piezoelectric load cells Kistler 9106A (full scale $330 \mathrm{kN}$ ) placed on the Reaction Wall supports.

- 3 Piezoelectric load cells Kistler 9106A (full scale $330 \mathrm{kN}$ ) interposed between the three aluminium plates and the main steel mass of the impactor.

- 2 Piezoelectric accelerometers Kistler 8202A (full scale $2000 \mathrm{~g}$ ) mounted on the steel mass of the impactor to evaluate its acceleration and its possible rotation.

- 1 Piezoelectric accelerometer Kistler 8202A (full scale $2000 \mathrm{~g}$ ) mounted on the central aluminium plate of the impactor to evaluate possible inaccuracies in the force measurement due to the plate inertia.

- 1 Piezoelectric pressure sensor Kistler $601 \mathrm{H}$ on the pressurized chamber of the blast actuator.

- 3 Photoelectric cells Pepperl Fuchs for triggering the data acquisition and for measuring locally the impactor velocity

\section{Experimental tests}

This section summarizes some results of the tests performed with the blast actuator on two reinforced concrete $(\mathrm{RC})$ beams in order to evaluate the performance of this equipment and its capability to simulate the effects of a blast explosion without using explosives.

The tests have been conducted on full scale RC beams of dimensions $250 \times 250 \times 2200 \mathrm{~mm}$, manufactured with standard materials and reinforcement. The designed compressive strength of the concrete is around $\mathrm{f}_{\mathrm{ck}}=$ $20 \mathrm{MPa}$ with a maximum aggregate size of $20 \mathrm{~mm}$. The reinforcement consists of 4 longitudinal deformed bars of $12 \mathrm{~mm}$ diameter and stirrups of $8 \mathrm{~mm}$ diameter spaced every $200 \mathrm{~mm}$, as shown in Fig. 7.

The test campaign has been conducted taking into account safety issues due to the elevated levels of energy stored and quickly released during the experiments. For this reason and taking into account the high complexity of the equipment under operation, the energy involved during the experiments has been increased gradually. The set-up is also covered by a safety box (a mixed aluminium and polycarbonate panel structure) that protects the operators conducting the experiment from accidental debris.

The first three tests have been conducted on the same specimen with an increasing impacting velocity, and thus several of the testing parameters (especially the specimen damage state) are not well defined. Although these tests 


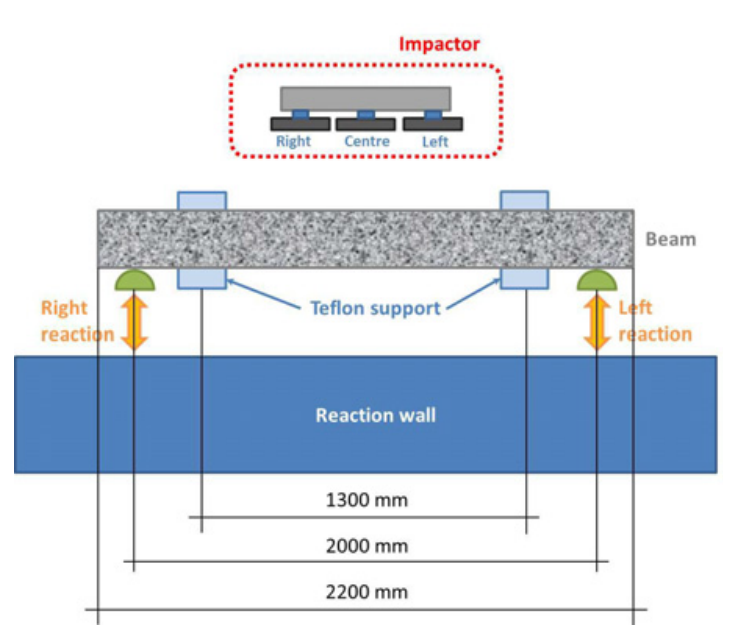

Figure 8. Schematic top view of the geometry of the experiments.

do not have a high scientific value, they have allowed a better knowledge of the equipment performance and of the test evolution. The tests have been performed by storing energy only in the actuator spring and a maximum velocity of about $12 \mathrm{~m} / \mathrm{s}$ has been reached. Following this test, the column has been judged to be overly damaged to continue with additional tests on it.

On the contrary, the last test presented in this paper can be considered to have a greater scientific value even though a series of experimental setbacks have occurred (the acquisition of piezoelectric sensors failed for a bug of the transient recorder). The test has been performed by employing the energy of the springs and of pressurized nitrogen in order to increase the impact velocity of the mass (about $17 \mathrm{~m} / \mathrm{s}$ ). The beam has been seriously damaged and patterns of a shear failure mode have appeared (also typical of blast load failure).

A first analysis of the recorded data has been made and some results in the form of images and diagrams are presented in the following. Figure 8 provides a general picture of the geometry of the impactor, the beam specimen and its supports, which helps in understanding the diagrams produced below for each one of the tests. Figure 9 presents a selection of representative frames of the high-speed photo-sequence captured during the last test with the high speed camera placed perpendicularly above the specimen.

The impacting mass hits normally the RC beam (frames 1 and 2) and forces it to bend, as shown in the frames 3 and 4. For the specific test, due the nonsymmetric behaviour of the specimen, the beam mainly fails in the left part as underlined by the rotation of the impacting mass (frame 4). In addition to the qualitative information that can be deduced by the high speed camera photo sequence (type of damage, possible rebounds, impact misalignments, etc.), special analyses have been employed for these optical data to obtain quantitative measures.

Specifically, two different algorithms have been applied:

- a fast tracking algorithm [3] that returns an accurate measure (in the order of $0.01 \mathrm{~mm}$ ) of the position of

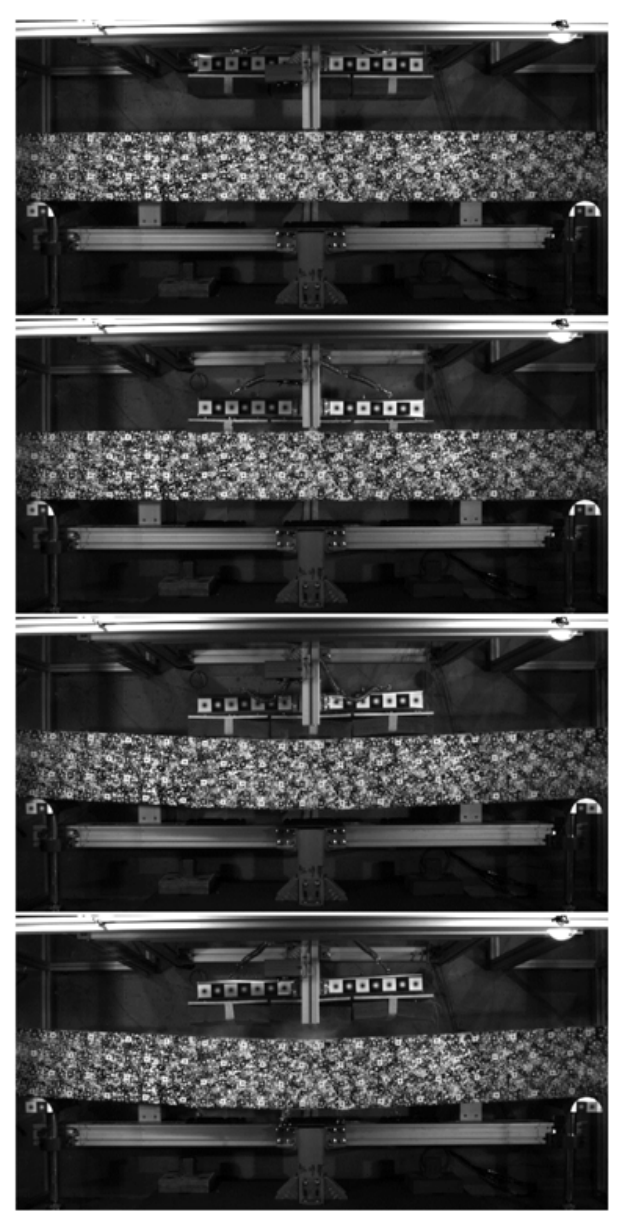

Figure 9. High speed photo sequence; time $=14 \mathrm{~ms}, 24 \mathrm{~ms}$, $34 \mathrm{~ms}$ and $40 \mathrm{~ms}$.

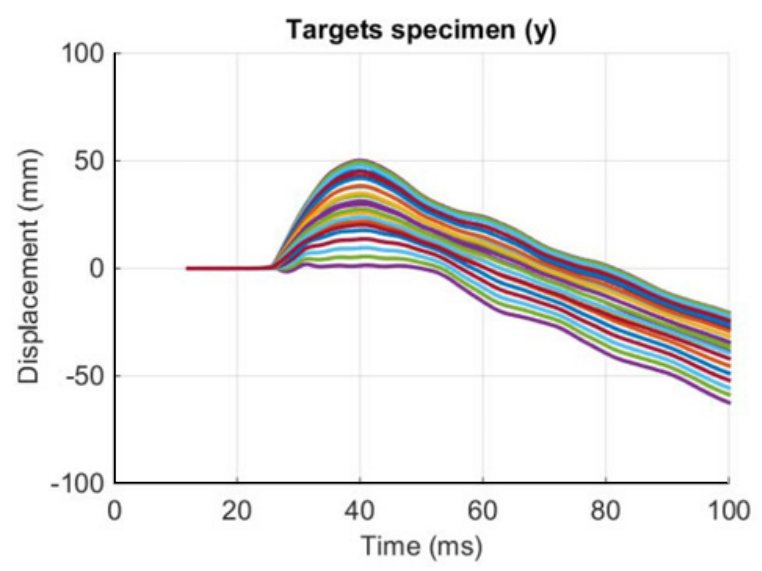

Figure 10. Beam target displacements (along impact direction).

the particular Gaussian grayscale targets applied to the column and to the impacting mass (Fig. 9);

- a full-field technique, the so called optical flow algorithm [4], that returns the displacement of each image pixel (with less precision compared with the previous algorithm), useful to study the evolution of the fracture paths during the test.

Figure 10 shows an example of quantitative data extracted with the first of the algorithm described above. The beam target displacements (along the impact direction) have 


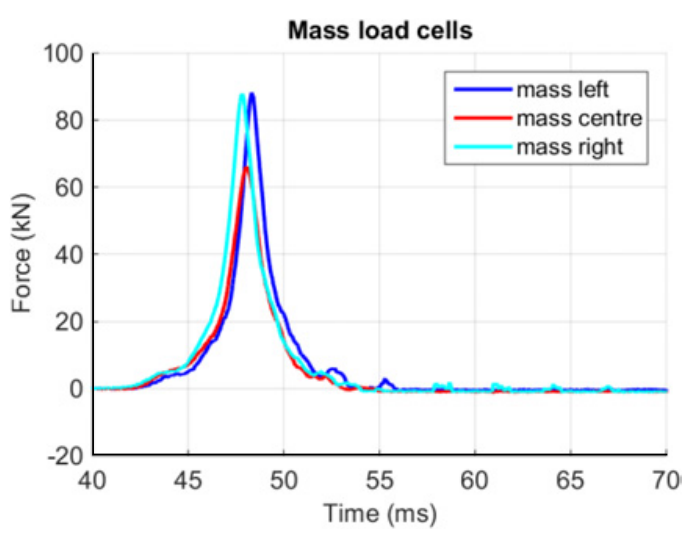

Figure 11. Mass load cell signals.

been presented. The impact starts approximatively at $22 \mathrm{~ms}$ and the maximum deflection occurs at $40 \mathrm{~ms}$ as underlined by the graph (the contact between the mass and the specimen lasts less than $8 \mathrm{~ms}$ ). Obviously the curves that present the greater deflections are related to targets located in the central part of the column. After $40 \mathrm{~ms}$ the elastic unloading of the damaged column starts, which causes the rebound of the specimen in the direction opposite to the impact. At $100 \mathrm{~ms}$ the bending oscillations due to the impact are substantially finished and the displacements of the targets are representative of the damaged shape of the beam. A permanent deflection of about $50 \mathrm{~mm}$ has been reached in the central part of the beam.

For what concerns the piezoelectric sensors placed on the impacting mass, Fig. 11 shows the signals recorded during the last experiment conducted on the first beam (the specimen was already damaged by previous experiments performed at low impact velocities). The direct measures of the impacting forces are totally noiseless and no ringing phenomena of the transducers occur in the duration of the test. In addition it is possible to observe that the force transmitted by the central plate is lower compared with those of the two lateral plates due to the pre-existing damage in the specimen. In any case the peak load and the duration of the pulses generated by the equipment are comparable with the experimental data obtained from experiments conducted with explosives.

Figure 12 shows the impulses related to Fig. 11. As expected, the signal derived by the central plate is lower compared with the two lateral ones.

\section{Numerical simulations}

Finite element analysis has been performed, in order to verify the experimental results and to ameliorate the final configuration of the apparatus. The geometrical model presented in Fig. 8 has been discretised, as depicted in Fig. 13, with finite elements via the CAST3M code [5]. The numerical calculations have been performed with the EUROPLEXUS explicit finite element code [6]. Hexahedral solid elements with eight nodes, one integration point and linear displacement interpolation have overall been preferred.

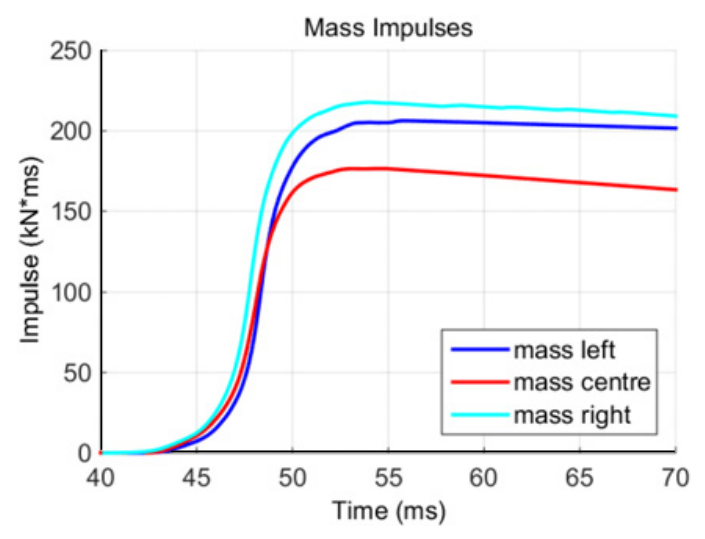

Figure 12. Impulses applied by three plates.

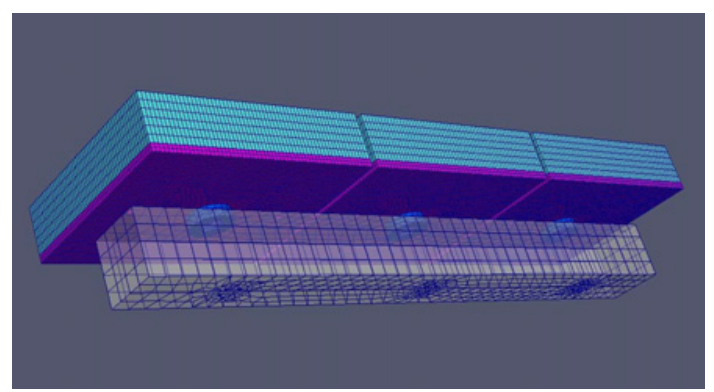

Figure 13. Finite element model of the impacting mass.

Different element types and sizes are employed in the representation of the impacting mass. For the massive steel part a relatively coarse mesh has been used since it will not undergo high strains (cubic edge size $20 \mathrm{~mm}$ ). For the load cells, the aluminium plates and the polyurethane foam finer meshes have been used with an average cubic edge of $5 \mathrm{~mm}$.

The concrete beam has been discretised with cubic finite elements of $20 \mathrm{~mm}$ edge size, while the reinforcement has been modelled with 1-D bar elements. The bonding between the concrete and the reinforcement has been modelled with a procedure that links the displacement of the nodes belonging to the continuum-like elements of concrete, with those of the bar-like elements of steel.

For the massive steel part of the impactor, the load cells and the beam reinforcement a Von Mises material law has been employed. Elasto-plasticity is implemented via a radial return algorithm and isotropic hardening is included. For the representation of the aluminium, a perfectly plastic Von Mises material law has been used. The concrete part has been introduced via a three-invariant cap model with mixed hardening and isotropic damage that contains also the strain-rate effect [6]. Finally, for the elastic foam a hyperelastic material law has been used, based on a strain energy equation [7]. The energy equation represents the stress-strain curve, with several parameters derived from material tests. The parameters have been defined after a best-fit procedure of the given stress-strain curve. Table 1 presents the mechanical properties (density, Young modulus, Poisson ratio) of the metallic and concrete parts 
Table 1. Mechanical properties used for the numerical model.

\begin{tabular}{|l|l|l|l|}
\hline & $\rho\left[\mathrm{kg} / \mathrm{m}^{3}\right]$ & $E[\mathrm{GPa}]$ & $v$ \\
\hline Steel & 7800 & 210 & 0.30 \\
\hline Aluminium & 2800 & 70 & 0.35 \\
\hline Load cell & 6488 & 131 & 0.30 \\
\hline Concrete & 2400 & 30 & 0.21 \\
\hline
\end{tabular}

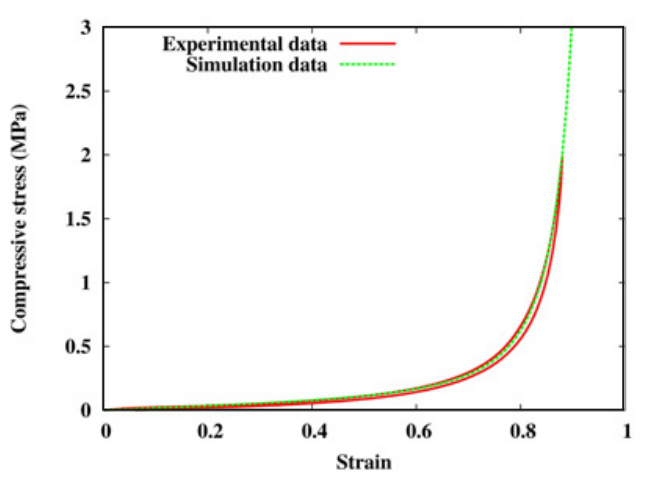

Figure 14. Stress-strain curve for the polyurethane foam.

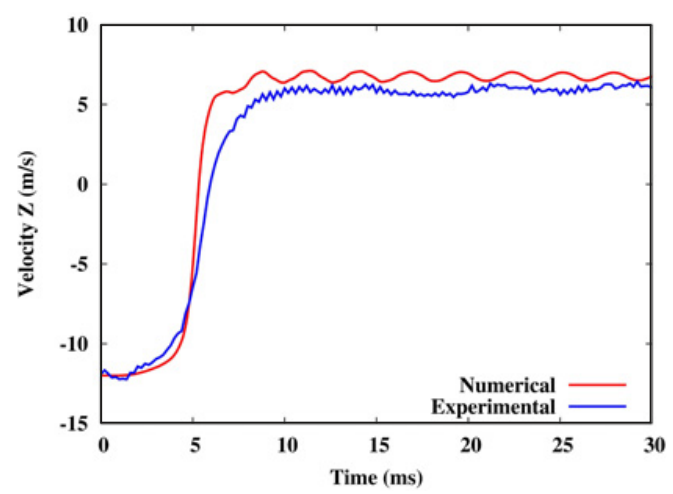

Figure 15. Velocity of the impactor.

of the structure, while Fig. 14 presents the stress-strain curve for the polyurethane foam (both experimental and numerical).

The impactor is moving with an initial velocity until it collides with the specimen. The pinball method $[8,9]$ has been used for the contact between the impacting mass and the specimen. The RC beam has been blocked in the vertical direction for all the points lying on the support lines.

Figures 15 and 16 present the comparison between the experimental and numerical results concerning the velocity and the displacement of the impactor in one of the initial tests. As seen, there is a relatively good agreement between the two approaches.

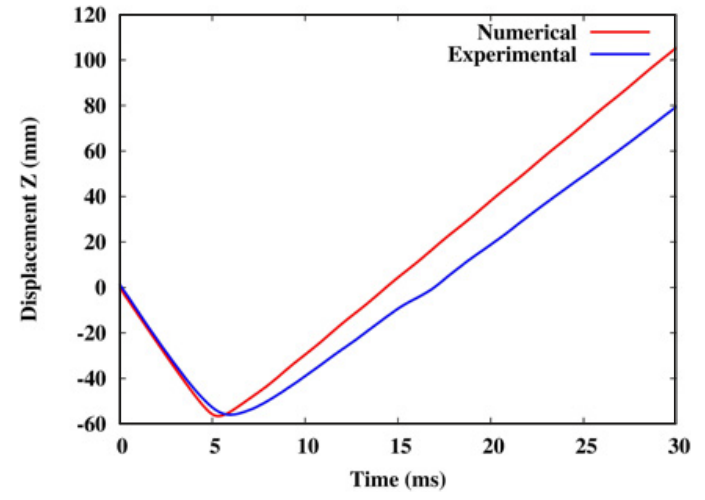

Figure 16. Displacement of the impactor.

\section{Conclusions}

Towards developing a blast simulator, a fast, spring-gas driven actuator and an innovative impactor have been constructed. Their performance has been assessed through appropriately instrumented tests. The results obtained from these tests demonstrate that the developed blast actuator is capable of reproducing through impact the required pressure levels to bring real structural members (reinforced concrete beams) to failure. Numerical simulations are also conducted, successfully modelling the experiments and pointing to improvements in the performance of the apparatus.

\section{References}

[1] G.A. Hegemier, F. Seible, T. Rodriguez-Nikl, K. Arnett, Blast Mitigation of Critical Infrastructure Components and Systems, FIB Proceedings of the 2nd International Congress, June 5-8 2006, Naples, Italy, paper ID: 11-2

[2] M. Peroni, G. Solomos, B. Viaccoz, Blast Simulator project: Performance assessment and preliminary tests, Report JRC 86864

[3] P. Caperan, M. Poljansek, E. Gutierrez, S. Primi, C. Paulotto, Comp. Struct. 94, 2012

[4] C. Zach, T. Pock, H. Bischof, A Duality Based Approach for Realtime TV-L ${ }^{1}$ Optical Flow, Pattern Recognition, Springer, 2007

[5] http://www-cast3m.cea.fr

[6] EUROPLEXUS User's manual, 2015; http:// www-epx.cea.fr

[7] S. Kolling. P.A. Du Bois. D.J. Benson and W.W. Feng. Comp. Mech., 40:885-899, 2007

[8] T. Belytschko, M.O. Neal, Int. J. Num. Meth. Engng., 31:547-572, 1991

[9] F. Casadei, A general Impact-Contact method based on hierarchic pinballs for the Europlexus software system, Report JRC I.03.176 